\title{
The Role of Amrinone in Potential Heart Transplant Patients With Pulmonary Hypertension
}

\author{
G. Michael Deeb, MD and Steven F. Bolling, MD
}

\begin{abstract}
Orthotopic heart transplantation is contraindicated in patients with pulmonary hypertension and an elevated pulmonary vascular resistance. In an attempt to make otherwise unacceptable patients possible candidates for heart transplantation, amrlnone was administered intravenously to 27 individuals with a transpulmonary gradient and pulmonary vascular resistance in the abnormal range. Twentyfour of 27 patients (89\%) responded positively. Twenty-one of 27 (78\%) went on to transplantation and 20 of 21 (95\%) survived the procedure. A second study compared amrinone therapy with conventional therapy in 38 potential transplant candidates with pulmonary hypertension. Amrinone was more effective in reducing pulmonary hypertension than conven-
\end{abstract}

$\mathbf{P}$ ULMONARY hypertension (PHT) has been a significant clinical problem since the inception of orthotopic heart transplantation. It quickly became apparent that heart transplant recipients with underlying PHT (pulmonary arterial systolic pressures greater than $50 \mathrm{~mm} \mathrm{Hg}$ ) had severe difficulty being weaned from cardiopulmonary bypass at the time of their transplantations, secondary to right-sided heart failure. Isoproterenol, a $\beta$-agonist, is generally administered during the weaning process to stimulate the ncwly transplanted heart and to initiate vigorous ventricular contraction. In the presence of PHT, the right ventricle will begin to distend as weaning from cardiopulmonary bypass proceeds, and contractility will markedly diminish. The right ventricle will eventually overdistend and cease contracting. Ultimately, the patient will become hypotensive and have minimal organ perfusion. Various techniques have been used to avoid this poor outcome, including the administration of isoproterenol as an infusion into the right atrium and a simultaneous epinephrine infusion into the left atrium. This modality of therapy would potentially increase the systemic blood pressure but would do little or nothing for the overall cardiac output and systemic perfusion. Alternative procedures for patients with significant PHT who are not orthotopic heart transplantation candidates include heart-lung transplantation or the heterotopic heart transplant procedure. Studies of pulmonary hemodynamics separated patients into certain distinct categories. Heart-lung tional therapy with high-dose diuretics, digitalis, and captopril (86\% v 63\%). Survival rate of those awaiting transplantation was also significantly higher in the amrinone group $(91 \% \vee 63 \%)$. Although the protocol for comparing the two regimens does not allow for extrapolation of the results (amrinone was administered in-hospital under close monitoring, whereas conventional therapy was self-administered at homel, the findings confirm the clinical impression that amrinone seems more effective and safer than conventional therapy in the treatment of potential heart transplant patients with pulmonary hypertension.

(C) 1989 by W.B. Saunders Company.

transplantation was reserved for those patients with primary PHT or fixed PHT, secondary to left-to-right shunt and the diagnosis of Eisenmenger's syndrome.

\section{PULMONARY DYNAMICS}

Not all patients with high pulmonary pressures suffered disastrous fates with orthotopic heart transplant; some were successfully weaned off bypass quite easily and recovered remarkably well. In an effort to identify the patients who would have difficulty, attention was focused on pulmonary hemodynamics.

Patients with elevated pulmonary pressures secondary to heart failure without a left-to-right shunt were the remaining group that needed to be subdivided further. These prospective heart transplant candidates fell into two groups, those patients with responsive pulmonary vasculature who could accept an orthotopic transplant and those with nonresponsive hemodynamics, who would best be accommodated by a heterotopic procedure. Kormos et al ${ }^{1}$ noted that the two most significant indicators of outcome in orthotopic heart transplantation were the transpulmonary gradient (TPG) and the pulmonary vascular

From the Section of Thoracic Surgery, University of Michigan Medical Center, Ann Arbor, MI.

Address reprint requests to G. Michael Deeb, MD, University of Michigan Medical Center, Ann Arbor, MI 48109.

(c) 1989 by W.B. Saunders Company. 0888-6296/89/0306-2007\$03.00/0 
resistance (PVR). It was noted that when a patient's TPG (pulmonary arterial mean pressure minus pulmonary capillary wedge pressure) was greater than $15 \mathrm{~mm} \mathrm{Hg}$ and the PVR was greater than 5 Wood's units (WU), the operative mortality with orthotopic heart transplantation was approximately $25 \%$, secondary to acute rightsided heart failure. ${ }^{1}$ Kirklin et $\mathrm{al}^{2}$ verified these findings using analysis of variance for risk factors in 61 orthotopic heart transplant patients and showed that elevated PVR caused a significant increase in the perioperative mortality. Therefore, efforts were directed to identifying patients with elevated TPG and/or PVR and targeting therapy to reduce the TPG to less than $15 \mathrm{~mm}$ $\mathrm{Hg}$ and the PVR to less than 5 WU.

It was decided that the majority of patients with elevated pulmonary hemodynamics secondary to primary congestive heart failure had increases in their pulmonary pressures, secondary to a high left ventricular end-diastolic pressure. Therefore, decreasing the systemic afterload should have ultimately reduced the pulmonary pressures and vascular resistances in these patients. Accordingly, at the time of rightsided heart catheterization, a patient with a pulmonary arterial systolic pressure greater than $50 \mathrm{~mm} \mathrm{Hg}$ and a TPG greater than $15 \mathrm{~mm} \mathrm{Hg}$ or a PVR greater than $5 \mathrm{WU}$ should undergo immediate systemic afterload reduction with intravenous sodium nitroprusside. If the patient responds to this maneuver with a decrease in the TPG to less than $15 \mathrm{~mm} \mathrm{Hg}$ and/or the PVR to less than $5 \mathrm{WU}$, then the patient should be considered a responder and placed on oral afterload reduction therapy. However, a large number of patients do not immediately respond with decreases in their TPG and PVR into an acceptable range and therefore are not considered candidates for orthotopic heart transplantation. It is conceivable that patients do not immediately respond because they have had chronic elevation of pressures caused by heart failure; they may require prolonged therapy.

\section{AMRINONE THERAPY}

Amrinone (Inocor, Winthrop, New York, NY) increases inotropy secondary to phosphodiesterase inhibition and causes smooth muscle relaxation and vasodilation. It seems to be an excellent drug for reducing the PVR in those patients who do not respond to sodium nitroprusside. Eichorn et al $^{3}$ reported that amrinone reduces right ventricular afterload and causes an increase in cardiac index and right ventricular systolic performance with a significant decrease in pulmonary end-systolic pressure. Therefore, they studied 27 patients who, on right-sided heart catheterization, had TPG greater than 15 $\mathrm{mm} \mathrm{Hg}$ and PVR greater than $5 \mathrm{WU}$ and were unresponsive to a challenge with sodium nitroprusside. These patients were thought to have pulmonary hypertension secondary to congestive heart failure, that was unresponsive to immediate left ventricular unloading; therefore, they were thought to be good candidates for a trial with prolonged intravenous amrinone therapy. ${ }^{4}$

The patients were admitted to the intensive care unit, where they underwent loading with $0.75 \mathrm{mg} / \mathrm{kg}$ of intravenous amrinone over a 15 minute period, and subsequently received a continuous infusion beginning at $2.5 \mu \mathrm{g} / \mathrm{kg} / \mathrm{min}$, titrating the dose up to a maximum of 20 $\mu \mathrm{g} / \mathrm{kg} / \mathrm{min}$ with a goal of achieving a pulmonary arterial systolic pressure less than $50 \mathrm{~mm} \mathrm{Hg}$, a TPG less than $15 \mathrm{~mm} \mathrm{Hg}$, and a PVR of less than 5 WU. Pulmonary and systemic hemodynamics were continously monitored, as were serum electrolytes and renal and hepatic functions. Serum platelet levels were also followed. After 48 hours, the systemic and pulmonary arterial catheters were removed, and the patients were recatheterized on a weekly basis. Twenty-four of 27 previously unresponsive pulmonary hypertensive patients $(89 \%)$ responded to this modality of therapy with a decrease in their pulmonary indices into a range acceptable for orthotopic heart transplantation. The average dose of amrinone was 9 $\mu \mathrm{g} / \mathrm{kg} / \mathrm{min}$. Patients required a mean of 3 days from the initiation of therapy until the target goals were attained. Patients who did not respond within the first week never responded subsequently. Twenty-one of the 24 patients who responded were fortunate enough to receive donor hearts and underwent orthotopic heart transplantation. Twenty of the 21 patients (95\%) who received transplants survived the procedure and were discharged.

The one patient who did not survive the procedure died of acute right-sided heart failure. This patient had difficulty being separated from cardiopulmonary bypass, necessitating mechani- 
cal assistance. The patient was an 8-year-old girl who had congestive cardiomyopathy associated with pulmonary hypertension. She entered the amrinone trial and responded to therapy with a decrease in her pulmonary parameters. Intravenous amrinone was discontinued, and she was discharged; it was planned to recatheterize her biweekly. Ten days after her discharge a donor organ became available, and at the time of her readmission her pulmonary pressures and resistance were, once again, unacceptable. She was again given amrinone, but did not respond immediately, and underwent orthotopic heart transplantation, but did not survive. Therefore, therapy has been continued on all subsequent patients until transplantation.

It was concluded that intravenous amrinone was an adequate drug for reducing pulmonary pressures and vascular resistance into an acceptable range for orthotopic heart transplant in these previously unresponsive patients, as noted by the $89 \%$ response rate. It was also felt that this was an effective modality of therapy since the perioperative mortality was reduced from $25 \%$ to $5 \%$.

\section{Therapy While Awaiting Transplantation}

However, prolonged intravenous amrinone is not practical because of the long wait for donor organs. Currently at this institution the average wait for a donor heart is 8 to 12 months. Since continuous in-hospital intravenous therapy is unreasonable for such a length of time, a more conventional medical regimen was initiated, including oral digoxin at $0.25 \mathrm{mg} / \mathrm{d}$, an oral angiotensin-converting enzyme inhibitor (captopril), and high-dose oral diuretics. Sixteen patients, who underwent standard right heart catheterization for pretransplant workup, met the protocol criteria for unacceptable pulmonary hemodynamics, and were not immediately respon- sive to left ventricular unloading with sodium nitroprusside received conventional therapy. Table 1 shows the drug regimens of the patients with pulmonary hypertension who received conventional therapy. All 16 patients were on furosemide, (average of $360 \mathrm{mg} / \mathrm{d}$ ), with 9 patients also on metolazone $(10 \mathrm{mg} / \mathrm{d})$ and an additional 5 patients on bumetanide $(5 \mathrm{mg} / \mathrm{d})$. Ten of 16 patients $(63 \%)$ were responsive to this therapy. There was an average of 8 days from the onset of therapy until the patients reached their desired hemodynamic parameters. Suitable donor organs were obtained for 7 of the 10 responding patients, and they underwent successful transplantation. Of the 16 patients treated with conventional therapy, 6 died suddenly at home $(63 \%$ survival awaiting transplant).

\section{Amrinone Versus Conventional Therapy}

Comparison of patients treated with conventional therapy with an age-matched group from the amrinone patient pool was undertaken. ${ }^{5}$ Twenty-two of the 27 previously discussed patients were included in the age-matched amrinone group. There was no significant difference in the rate of response between the two groups. Nineteen of 22 patients $(86 \%)$ in the amrinone group responded, and 10 of 16 patients $(63 \%)$ in the conventional therapy group responded $(P=0.053)$. Comparing the survival rate in the two groups, 20 of 22 patients in the amrinone group survived $(91 \%)$, whereas only 10 of 16 patients in the conventional group survived (63\%). This difference was significant at the $P<0.05$ level. However, it may not be fair to compare these two groups because the amrinone group received intravenous therapy in the hospital while being monitored, whereas the conventional group was at home. The causes of death while awaiting transplant were different between the two groups. Two patients in the amrinone group died of

Table 1. Drug Regimens of Pulmonary Hypertension Patients

\begin{tabular}{|c|c|c|c|c|c|}
\hline \multicolumn{3}{|c|}{ Amrinone Therapy } & \multicolumn{3}{|c|}{ Conventional Therapr } \\
\hline Name & Dose & $\begin{array}{c}\text { No. of } \\
\text { Patients }\end{array}$ & Name & Dose & $\begin{array}{c}\text { No. of } \\
\text { Patients }\end{array}$ \\
\hline Furosemide & $40 \mathrm{mg} / \mathrm{d}$ & 9 & Furosemide & $360 \mathrm{mg} / \mathrm{d}$ & 16 \\
\hline Digoxin & - & - & Digoxin & $0.250 \mathrm{mg} / \mathrm{d}$ & 16 \\
\hline Captopril & - & - & Captopril & $37.5 \mathrm{mg} / \mathrm{d}$ & 11 \\
\hline Metolazone & $5 \mathrm{mg} / \mathrm{d}$ & 2 & Metolazone & $20 \mathrm{mg} / \mathrm{d}$ & 9 \\
\hline Bumetanide & - & - & Bumetanide & $6 \mathrm{mg} / \mathrm{d}$ & 5 \\
\hline Amrinone & $0.75 \mathrm{mg} / \mathrm{kg}$ loading $9 \mu \mathrm{g} / \mathrm{kg} / \mathrm{min}$ & 22 & Amrinone & - & - \\
\hline
\end{tabular}


progressive left ventricular failure unresponsive to amrinone therapy, whereas the outpatients receiving conventional therapy died suddenly, quite possibly from dysrhythmias induced by the high dose of diuretics and digoxin.

Analysis of amrinone and conventionally treated patients showed no significant differences in the pretreatment pulmonary and systemic hemodynamic parameters. Comparison of the pretreatment versus posttreatment mean pulmonary and systemic parameters in the group receiving amrinone therapy showed there were significant reductions in pulmonary pressures, TPG, and PVR. There was also a significant increase in the cardiac output with no significant change in the heart rate or the systemic arterial or venous pressures. The systemic vascular resistance fell, but not significantly (Table 2). Comparison of pretreatment versus posttreatment mean hemodynamic values for patients receiving conventional therapy showed both the pulmonary and the systemic hemodynamics significantly changed (Table 3).

Therefore, it was concluded that the desired pulmonary changes with the conventional group were secondary to systemic hemodynamic changes, but that the changes in the amrinonetreated group were quite possibly caused by a primary vasodilation of the pulmonary vasculature. To further support this concept, comparison of post-therapy parameters between the two different treatment groups showed a significant

Table 2. Amrinone Therapy: Prehemodynamics Versus Posthemodynamics (N $=22$ )

\begin{tabular}{lccc}
\hline & Prehemodynamics & $P$ Value & Posthemodynamics \\
\hline BUN (mg/dL) & $28.8 \pm 2.3$ & NS & $30.2 \pm 1.6$ \\
Creatinine & & & \\
$\quad(\mathrm{mg} / \mathrm{dL})$ & $1.3 \pm 0.06$ & NS & $1.39 \pm 0.07$ \\
PAM (mm Hg) & $44.3 \pm 1.6$ & $<0.0001$ & $29.0 \pm 1.5$ \\
PCWP (mm Hg) & $25.2 \pm 1.1$ & $<0.0001$ & $18.5 \pm 1.2$ \\
TPG (mm Hg) & $19.0 \pm 1.2$ & $<0.0001$ & $10.9 \pm 0.9$ \\
CO (L/min) & $3.5 \pm 0.3$ & $<0.0015$ & $4.56 \pm 0.3$ \\
PVR (WU) & $5.87 \pm 0.4$ & $<0.0001$ & $2.49 \pm 0.2$ \\
MAP (mm Hg) & $79.0 \pm 3.4$ & NS & $80.3 \pm 3.0$ \\
CVP (mm Hg) & $12.5 \pm 1.9$ & NS & $8.3 \pm 1.8$ \\
SVR (WU) & $20.8 \pm 2.1$ & NS & $16.4 \pm 1.2$ \\
\hline
\end{tabular}

Abbreviations: BUN, blood urea nitrogen; PAM, pulmonary arterial mean pressure; PCWP, pulmonary capillary wedge pressure; TPG, transpulmonary gradient; PVR, pulmonary vascular resistance; MAP, mean arterial pressure; CVP, central venous pressure; SVR, systemic vascular resistance.
Table 3. Conventional Therapy Prehemodynamics Versus Posthemodynamics ( $N=16$ )

\begin{tabular}{lrlc}
\hline & Prehemodynamics & $P$ Value & Posthemodynamics \\
\hline BUN (mg/dL) & $27.7 \pm 3.5$ & NS & $30.2 \pm 2.5$ \\
Creatinine $(\mathrm{mg} / \mathrm{dL})$ & $1.4 \pm 0.1$ & NS & $1.39 \pm 0.09$ \\
PAM $(\mathrm{mm} \mathrm{Hg})$ & $46.2 \pm 1.8$ & 0.0001 & $32.2 \pm 2.2$ \\
PCWP (mm Hg) & $27.7 \pm 1.8$ & 0.002 & $18.9 \pm 1.8$ \\
TPG (mm Hg) & $18.2 \pm 1.0$ & 0.003 & $13.4 \pm 1.0$ \\
CO (L/min) & $3.6 \pm 0.2$ & 0.03 & $4.2 \pm 0.15$ \\
PVR (WU) & $5.1 \pm 0.4$ & 0.003 & $3.3 \pm 0.3$ \\
MAP (mm Hg) & $85.0 \pm 3.6$ & 0.03 & $75.7 \pm 2.0$ \\
CVP (mm Hg) & $11.7 \pm 1.9$ & 0.017 & $5.7 \pm 1.3$ \\
SVR (WU) & $21.2 \pm 1.3$ & 0.02 & $16.6 \pm 1.1$ \\
Weight (kg) & $84.6 \pm 3.6$ & NS & $78.9 \pm 3.4$ \\
\hline
\end{tabular}

Abbreviations: See Table 2.

difference only in the PVR. Although both groups showed significant reductions in PVR during the presetting versus postsetting, the decrease in the PVR in the amrinone group was significantly greater than that in the conventional group. This finding is in light of the fact that there was no significant difference in the PVR between the two groups prior to therapy.

\section{CONCLUSION}

Intravenous amrinone is an excellent therapy for patients with pulmonary hypertension, secondary to congestive heart failure $(90 \%$ response rate). Patients who respond to amrinone therapy can subsequently undergo orthotopic heart transplantation with a $<5 \%$ operative risk. The time of onset from initiation of therapy until desired parameters were attained was 3 days, but this duration could be significantly shortened by increasing the loading dose and starting at a higher continuous intravenous infusion rate. Using more current loading protocols recommended by Hines and Levy would allow a much more rapid therapeutic blood level of amrinone to be achieved and could quite possibly shorten the duration of treatment [see Levy and Bailey, p 10, and Hines, p 24, in this issue ]. If higher doses of intravenous amrinone are given, the required duration of therapy decreases to 2 to 3 hours, and it may be possible to test patients for their responsiveness to amrinone on an acute basis in the catheterization laboratory. These patients could be discharged and treated again with amrinone at the time of readmission for transplantation. Currently, prolonged amrinone therapy is 
limited because it requires continual therapy, and the present wait for donor hearts ranges from 8 months to 1 year. Conversely, conventional therapy is more practical and has a reasonable success rate $(63 \%)$. Once the patients' parameters are acceptable for orthotopic heart transplantation, the perioperative success rate is excellent. However, the preoperative mortality rate is high with conventional therapy, believed to be second- ary to sudden death from dysrhythmias. Therefore, all the patients receiving conventional therapy are also being treated with amiodarone until the time of transplantation.

The ideal therapy may be an oral phosphodiesterase inhibitor that could be used in an outpatient setting, yet would show the excellent response rate seen with the intravenous form of amrinone.

\section{REFERENCES}

1. Kormos RL, Thompson M, Hardesty RL, et al: Utility of preoperative right heart catheterization data as a predictor of survival after heart transplantation. I Heart Transplant 5:391, 1986

2. Kirklin JK, Naften OC, McGiffin DC, et al: Analysis of morbid events and risk factors for death after cardiac transplantation. J Am Coll Cardiol 11:917-924, 1988

3. Eichorn EJ, Konstam MA, Weiland DS, et al: Differential effects of milrinone and dobutamine on right ventricular preload, afterload, and systolic performance in congestive heart failure secondary to ischemic or idiopathic dilated cardiomyopathy. Am J Cardiol 60:1329-1333, 1987

4. Bolling SF, Deeb GM, Crowley DC, et al: Prolonged amrinone therapy prior to orthotopic cardiac transplantation in patients with pulmonary hypertension. Transplant Proc 20:753-756, 1988

5. Deeb GM, Bolling SF, Guynn TP, et al: Amrinone versus conventional therapy in pulmonary hypertensive patients awaiting cardiac transplantation. Ann Thorac Surg (in press) 